\title{
An empirical study on mobile phone usage
}

\author{
Ali Mahmood Khan \\ Universität Bremen \\ Am Fallturm 1, TZi, D-28359 Bremen, Germany \\ akhan@tzi.de
}

\begin{abstract}
One of the major scientific undertakings over the past few years has been exploring the interaction between humans and machines in mobile environments. We have smart devices with high computational power. Some devices are also aware of context. For example, some mobile devices have built in GPS, light sensor and other detection devices like [1]. In this work, we will examine how mobile device could predict users' wishes regarding the push services like incoming mobile phone call. We conducted some experiments in order to get contextual data and then did analysis, a limited number of sensors were tagged to the user which were meant to detect certain characteristics of the environment the users were in, such as light sensor, temperature sensor, surrounding audio/noise level. Our results show that machine learning algorithms were able to classify the instances correctly with a high accuracy rate.
\end{abstract}

Sensor tool-kit/detection, adaptation, machine learning, user state, empirical studies

\section{INTRODUCTION}

Mobile phones are supposed to be carried around and their widespread use accounts for their presence in nearly every situation, however their alarm can be a real nuisance, but turning them off is no solution as people might want to receive urgent messages from senders. Therefore, callers can be grouped according to their relevance and profiles of availability can be specified. But all this has to be implemented, activated and changed manually by the user, who might find these activities too cumbersome to use them regularly. It would be desirable to have the user's situation and availability checked and classified automatically. In short, a mobile device should be able to adapt to the environment and control the device's settings. Such a system that would help users to avoid irrelevant calls without disturbing others should be available for all mobile phone users. In order to overcome the problem described above, a system can be implemented, using machine learning algorithms, which is aware of the availability of the user in everyday situations.

\section{RESEARCH QUESTION}

We want to prove that it is possible to determine the user's wish regarding receiving the incoming mobile calls by using some contextual information like surrounding temperature, noise and light intensity, user location like "indoors" or "outdoors", user's physical position like "sitting", "horizontal" and "vertical" and location of the mobile device like "pocket", "bag", "table" and "hand".

\section{MOTIVATION}

We want to figure out how to determine the user's wish regarding the incoming phone calls. We cannot rely completely on the mobile phone's scheduler where the users have to enter their schedules and appointments. We could take this information into account but we found some problems in this solution. What would happen if the user reschedules his meetings? What would happen, if the user forgot to mention them? What would happen, if the user has an impromptu meeting?

The bottom line is that we cannot absolutely rely on such a system, we need a system which should be adaptable, it should be able to learn from its context and the user's behaviour and it should be able to reduce the consciousness factor from human's daily life. A user should not be bothered to change his mobile device's ringer settings at any time. The mobile device should be aware of its context and adapt to it. Of course, every caller has some priorities, sometimes people want to receive calls only from selected people, sometimes they want to receive phone calls only from their families and sometimes they don't want to receive any phone call at all. We are interested to know whether user wants to receive a phone call or not? We do have some research papers, where the user carries some sensors on his body or device and the 
system detects the user's behaviour on the basis of input values and sets the mobile phone preferences[2],[3]. These systems work on predefined values, they do not adapt to the environment and they do not learn since they work on predefined inputs.

In our research, we conducted some experiments in order to determine whether user wants to receive a phone call or not? The testers had to carry a sensor box, which recorded the sensed information (light, audio and temperature). We also asked some questions from the users during the experiments in order to know about their activities and behaviours.

\section{RELATED WORK}

Integrating sensors with the mobile devices is not a new research field, a lot of research has been done in this area[2,3,4] etc. Researchers used several sensors for collecting the contextual information like light sensor[2,3], temperature sensor[2,3] and audio sensor[2,3] in order to determine the users' preferences regarding the mobile phone's push services. However, researchers have found ways to determine the users' preferences, but the issue is that these systems work only on the self assumed scenarios, learning is missing in their researches. We want our system to learn from the users' behavior and adopt it accordingly.

\section{HYPOTHESIS}

The contextual information measured by the light, temperature and audio sensor, user's physical activity and mobile device location, indicate whether user wants to receive a phone call or not

\section{EXPERIMENTAL SETUP}

We gave a sensor toolkit and hand-held device for a day to our testers and asked them to start the experiment from $9 \mathrm{AM}$ to $9 \mathrm{PM}$. We designed an application for the PDA, which used to ask some questions from testers during the experiment and meanwhile the sensor toolkit used to log sensed data into the device.

\subsection{Questionnaire form for PDA}

We implemented a small application for a handheld device, which interrupts the user every 30 minutes. For interruption, we employed the vibrator of the mobile device, which gives haptic feedback to the user (just like when you receive a call on your mobile device). The user needs to fill the following questionnaire form every 30 minutes as shown in Figure 1:

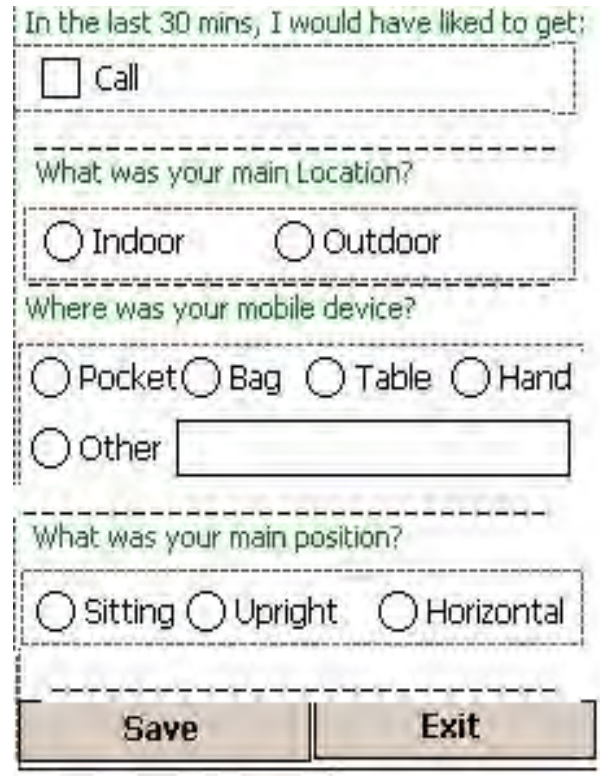

Figure 1: Questionnaire form

\subsection{Environmental Information}

We designed a circuit which used to log the light intensity, temperature level (in centigrade) and audio intensity during the whole experiment.

\section{HARDWARE SETUP}

We used Arduino board [5], light sensor, Temperature sensor, audio sensor and laptop. We used a breadboard with sticky cables in order to check the results. The Arduino board draws power from the laptops' battery; of course we could also use normal batteries for the arduino board instead of a heavy laptop. The reason being, since we were interested to gather data, for off line analysis, we need almost $5 \mathrm{MB}$ of space for environmental information. However the problem is that you cannot store this amount of data on an Arduino board. The Arduino board offers only 512 bytes, which is not enough for our experiments. So for this reason we used laptop as a data logger and power source for Arduino board. Of course, testers had to take care for laptop's battery while testing and it should be turned on during the experiment. We designed the following circuits.

\subsection{Light sensor}

The light sensor returns the integer value from 0 to 1024, 0 means black and 1024 means bright. We need to put it on the board in order to use it[6]. 


\subsection{Temperature sensor}

The temperature sensor returns an integer value that we converted into centigrade[7].

\subsection{Microphone sensor}

The microphone sensor returns the integer value from 0 to 1024, 0 means silent and 1024 means very loud. We need to put it on the board in order to use it[8].

\subsection{Sensor bag}

Firstly, we built a sensor bag (see figure: 5), and then put laptop and circuits with Arduino board in the shoulder bag. We stitched sensors on the shoulder bag's strap. We figured out, that these sensors should be on the shoulder, because, the shoulder is next to the ear and it will be useful for audio sensor. The light sensor should remain uncovered, otherwise, it will provide wrong data. Temperature sensor should also be in an open place in order to get some reliable data. This is why we stitched all sensors in one area, which will be hanged on the tester's shoulder. The bag's weight is $5 \mathrm{Kg}$ and we had some reservations that it would be little difficult for the testers due to long cables and its heavy weight.

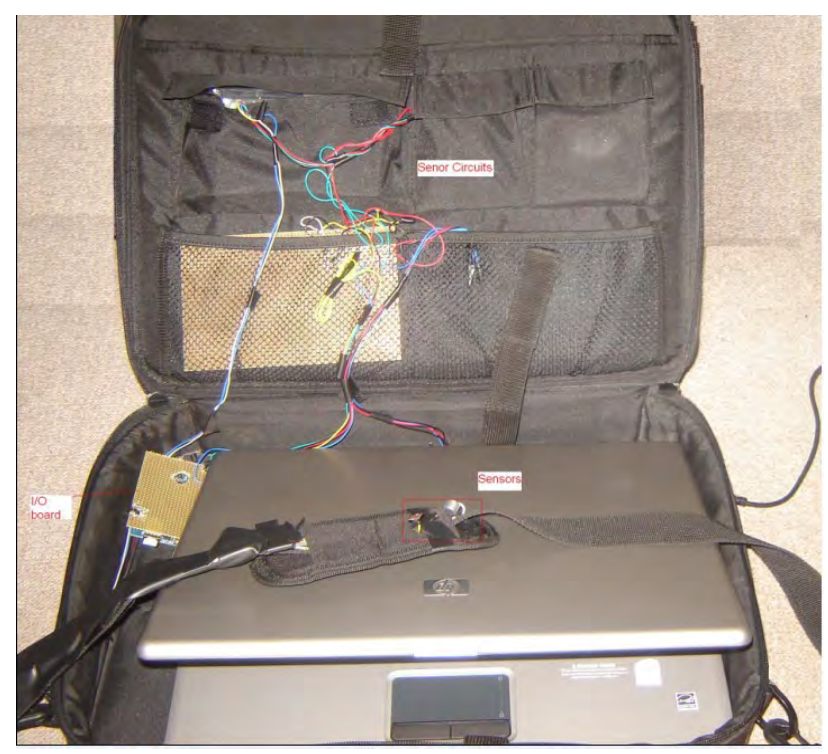

Figure 2: Sensor box

\subsubsection{Testers' feedback}

For our initial tests we recruited five testers. We We recruited five testers and asked them to carry shoulder bag and PDA. Following is the feedback that we got from the testers:

- Testers were uncomfortable and afraid due to long open cables.
- It was difficult for them to carry heavy laptop with sensors for 12 hours.

- Once laptop's battery was unplugged while carrying the laptop and we lost some data. In one instance the laptop fell down and one circuit was broken.

However, we could avoid this kind of a feedback by making sure that the battery won't be unplugged in the future and the circuits can be prevented from little accidents but we preferred to design a new sensor toolkit because we came to know after some discussions with the testers that our experiment setup was changing their daily routines, since; it was very difficult for them to deal with this kind of a setup.

- One tester, who usually commuted by bicycle, on the test day used car due to experiment.

- Some testers changed their schedule due to this setup.

- Some testers, who used to go to super market for shopping every evening, cancelled their shopping on the experiment day.

- Some testers did not go for jogging due to the setup.

This implies that we were not getting the right data, because our setup was indirectly changing the data. Testers were comfortable with PDA and even with the PDA application. Of course it was a little bit annoying for them to fill the form every 30 minutes, but it was not changing their daily routines. The only problem was in the laptop's size and its weight so we wanted to find another solution which would help us get rid of the laptop.

What was the importance of the laptop in our experiment? It was only providing the power to sensors, for which we could use, some external small batteries. It was also being used as a data logger with time stamp, because we were appending system's time in the environmental information $\mathrm{n}$ run time basis. Evidently, we were using the laptop only for the data logging purpose. we also thought about using a light weight laptop, but still, it did not solve the problem

\subsection{Sensor box}

Later, we designed a sensor box for our experimental setup, we took a box of $9 \times 12 \times 4 \mathrm{~cm}$ dimension and data logger (see figure: 3 ). We designed a circuit for all sensors, data logger, and rechargeable battery packs. Then we connected them with an arduino board. We made some slots for a battery charger, a power button, a reset button for sensors and for the memory slot. 


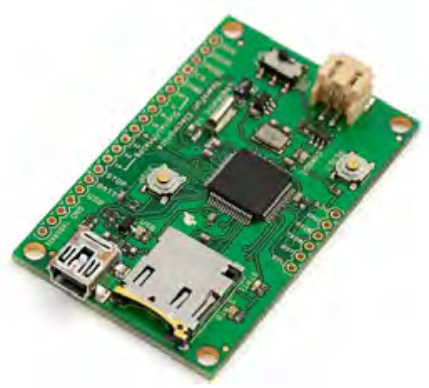

Figure 3: data logger [9]

We fixed everything in the box and enabled the connection of whole setup for the power button, the reset button and the battery charger. We passed the sensors through the box, for this purpose we used a .5 meter cable as you can see on Figure 4(a) and 4(b). We wrote a code for the Arduino board in order to get the sensed data from the sensors.

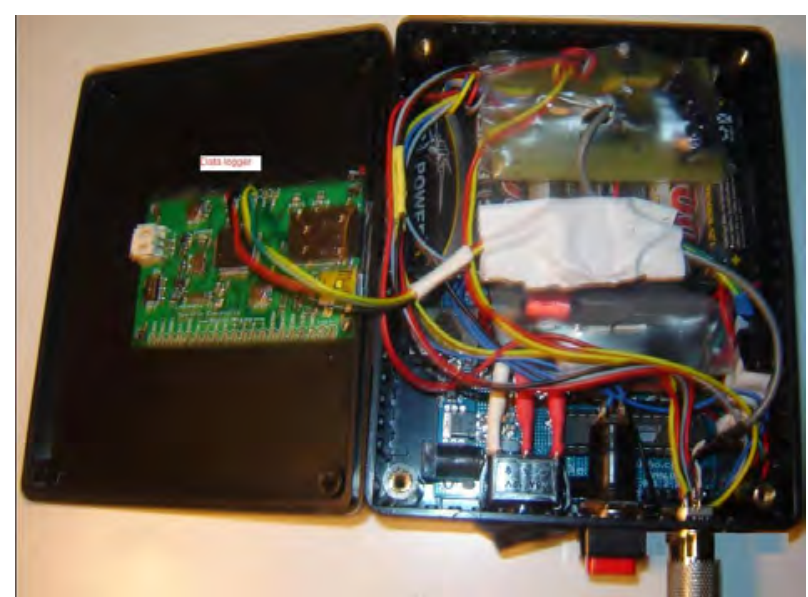

Figure 4(a): Sensor box-inside

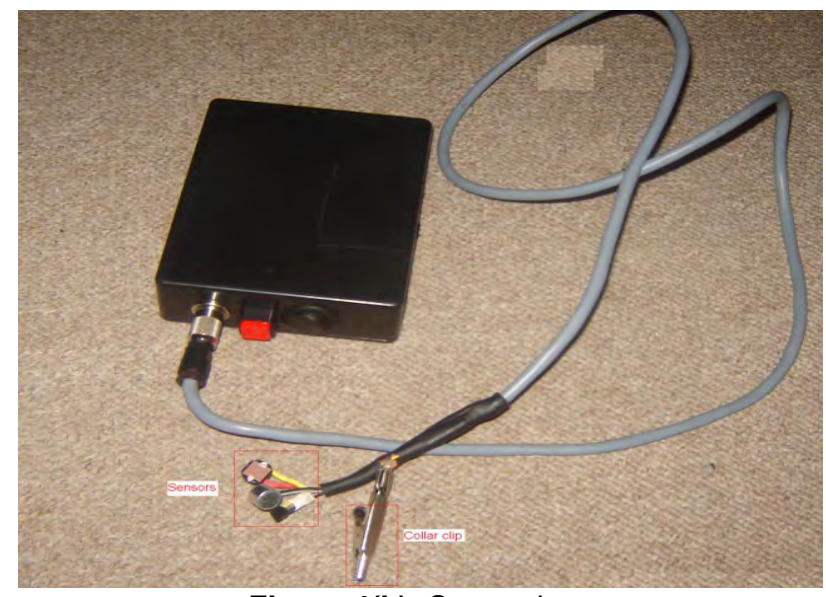

Figure 4(b): Sensor box
We got a very good feedback from the testers as compared to our first setup, they found a big difference in both setups.

\section{DATA GATHERING}

For our first setup, we recruited five testers but we discarded their data and for the second setup we recruited four testers. Basically, our testers were supposed to carry the sensor box with the PDA for 12 hours. Sensor box was logging the contextual information, data rate was one sample per second. PDA application used to interrupt testers in every 30 minutes and asked some questions, it was also logged by our system. At the end, we had (12X60X60) 43200 instances for each tester.

\subsection{Categorization of Data}

We got only numeric data from the sensed data. We categorized them in following orders:

Table 1: Categories for light intensity values

\begin{tabular}{|c|c|}
\hline Range & Category \\
\hline 0 to 349 & Dark \\
\hline 350 to 649 & Twilight \\
\hline 650 to 1023 & Light \\
\hline
\end{tabular}

Table 2: Categories for audio sensor's values

\begin{tabular}{|c|c|}
\hline Range & Category \\
\hline 0 to 399 & Low \\
\hline 400 to 499 & Medium \\
\hline 500 to 1023 & High \\
\hline
\end{tabular}

Table 3: Categories for temperature's data

\begin{tabular}{|c|c|}
\hline Range & Category \\
\hline 0 to 9 & Very cold \\
\hline 10 to 19 & Cold \\
\hline 20 to 29 & Normal \\
\hline 30 to 39 & High \\
\hline 40 to 1023 & Very high \\
\hline
\end{tabular}




\section{RESULTS}

At the end of the tests, we obtained the experimental data from the PDA application(main_location, mobile_device, main_position, phone_call) and sensed data from the sensor box(light_info, audio_info, temperature_info).We applied the decision tree and AODE (Averaged One-Dependence Estimators) classifier using weka toolkit(it is a software, where you can use built-in machine learning algorithms) [10] on the data set of each tester. Decision tree and AODE classifier correctly classify the instances with a good range of percentage.

Table 4: Overall classifier results

\begin{tabular}{|c|c|c|c|c|}
\hline & Minimum & Maximum & Average & SD \\
\hline $\begin{array}{c}\text { Decision } \\
\text { Tree }\end{array}$ & $84.28 \%$ & $100.00 \%$ & $90.37 \%$ & 7.6 \\
\hline AODE & $84.29 \%$ & $100.00 \%$ & $90.04 \%$ & 7.48 \\
\hline
\end{tabular}

Later, we divided our data from each tester into different chunks, with each chunk holding the data of one hour. Then we divided each chunk into two sub chunks of 30 minutes each. The first sub chunk was used for testing and the other for training. At the end we had 12 training data sets and 12 testing data sets for each tester. We applied decision tree classifier on the training data sets and built models for each data set. Later we applied the models on each testing data set in order to predict the user's wish regarding receiving the incoming call. We got (30X60) 1800 predictions for each testing data set. From each testing set the state with the highest prediction votes was chosen.

Table5: Predicted results for each tester (12 data sets)

\begin{tabular}{|c|c|c|c|c|}
\hline & Tester1 & Tester2 & Tester3 & Tester4 \\
\hline Min & $0.00 \%$ & $0.00 \%$ & $100.00 \%$ & $0.00 \%$ \\
\hline Max & $100.00 \%$ & $100.00 \%$ & $100.00 \%$ & $100.00 \%$ \\
\hline Average & $91.67 \%$ & $83.33 \%$ & $100.00 \%$ & $66.67 \%$ \\
\hline
\end{tabular}

Our system was able to predict $12 / 12$ times for the Tester3, because Tester3 never wanted to receive any phone call at all. Tester4 was in the same circumstances for few hours but he had different wishes in training and testing sessions.

\section{CONCLUSION}

In our research, we have gathered experimental data with sensors in order to get contextual information. We learned that we should be careful while designing the device for this kind of experiment; we should know whether our setup is influencing users' daily routines or not. This implies that it is important to log the users activity without disturbing them. We have shown that integration of diverse sensors is a viable approach to obtain contextual information in empirical research for a long duration. We could not get a lot of test data because it was difficult to get testers for this kind of a setup. If we had more data the results could have been better.

Contextual information like date and time should also be taken into account in future experiments because users' schedules depend on them. Also it would be interesting to observe the effect of conducting the tests on consecutive days on the prediction capabilities of our machine learning algorithm.

Despite having less test data, our results show that we have attempted to use this information to successfully predict the instances where a mobile phone user would be available to accept an incoming call.

\section{ACKNOWLEDGEMENT}

I am sincerely thankful to my supervisor, Prof. Dr. Michael Lawo, who helped me at every stage during my research. He helped me in clearing all my doubts and increased my knowledge through his valuable suggestions and inputs. I am very thankful to my testers, for voluntarily testing my setup.

\section{REFERENCES}

1. http://www.apple.com/iphone/specs.html; 12th February, 2011

2. Siewiorek, D., A. Smailagic, J. Furukawa, A. Krause, N. Moraveji, K. Reiger, J. Shaffer, and F. L. Wong. 2005. Sensay: A context-aware mobile phone. In Seventh IEEE International Symposium on Wearable Computers, 2003. Proceedings, 248249

3. Schmidt, A., K. A. Aidoo, A. Takaluoma, U. Tuomela, K. Van Laerhoven, and W. Van de Velde. 1999. Advanced interaction in context. In Handheld and Ubiquitous Computing: First International Symposium, Huc'99, Karlsruhe, Germany, September 27-29, 1999, Proceedings, 89. Springer.

4. Ballagas, R., F. Memon, R. Reiners, and J. Borchers. 2006. iStuff Mobile: prototyping interactions for mobile phones in interactive 
spaces. In Proc. PERMID, Workshop on Pervasive Mobile Interaction Devices at PERVASIVE.

5. http://www.arduino.cc/; 12th February, 2011

6.http://wiring.org.co/learning/basics/photoresistor.h tml ; 16th February, 2011

7. http://www.danielandrade.net/2008/07/05/temperat ure-sensor-arduino/ ; 12th February, 2011

8.

http://wiring.org.co/learning/basics/microphone.html ; 16th February, 2011

9.

http://www.sparkfun.com/commerce/product_info.p hp?products_id=8627; 12th Feb 2011

10. Mark Hall, Eibe Frank, Geoffrey Holmes, Bernhard Pfahringer, Peter Reutemann, Ian $\mathrm{H}$. Witten (2009); The WEKA Data Mining Software: An Update; SIGKDD Explorations, Volume 11, Issue 1. 